\title{
Living with dignity among Chinese cancer patients undergoing chemotherapy: a qualitative study
}

Jinnan XIAO, Carmen Wing Han CHAN, Ka Ming Chow

The Nethersole School of Nursing, Faculty of Medicine, The Chinese University of Hong Kong

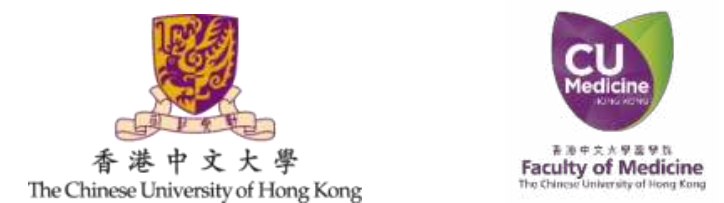

Introduction: Safeguarding the dignity for cancer patients has recently been gaining attention, whereas its development is constrained by the ambiguity construct of dignity. This study aims to describe dignity perceived by cancer patients receiving chemotherapy based on Dignity Model* and provide insights on promoting dignity conserving care in China.

Methods: A qualitative description design was conducted with face-to-face, semi-structured, individual interviews. Consecutive sampling of cancer patients receiving chemotherapy in a public hospital was recruited. The data was analyzed using the Framework Method analysis with both inductive and deductive approaches. Results: 15 cancer patients with age ranged from 28 to 66 years completed the interviews.

*: Chochinov, H. M., Hack, T., McClement, S., Harlos, M., \& Kristjanson, L. J. (2002). Dignity in the terminally ill: An empirical model. Soc Sci Med, 54(JUNE), 433-443.
Results (continuous): The summary of the categories, themes and subthemes is presented in the table.

Conclusions: Patients' sense of dignity can be affected by various issues, including illness, personal attitudes and behavior, and social environment. Culture and economics impact the construct of dignity hugely among Chinese population. Family-oriented approaches are recommended to create an environment where patients feel valued, respected and supported, helping cancer patients adapt to the illness changing process and maintain dignity.

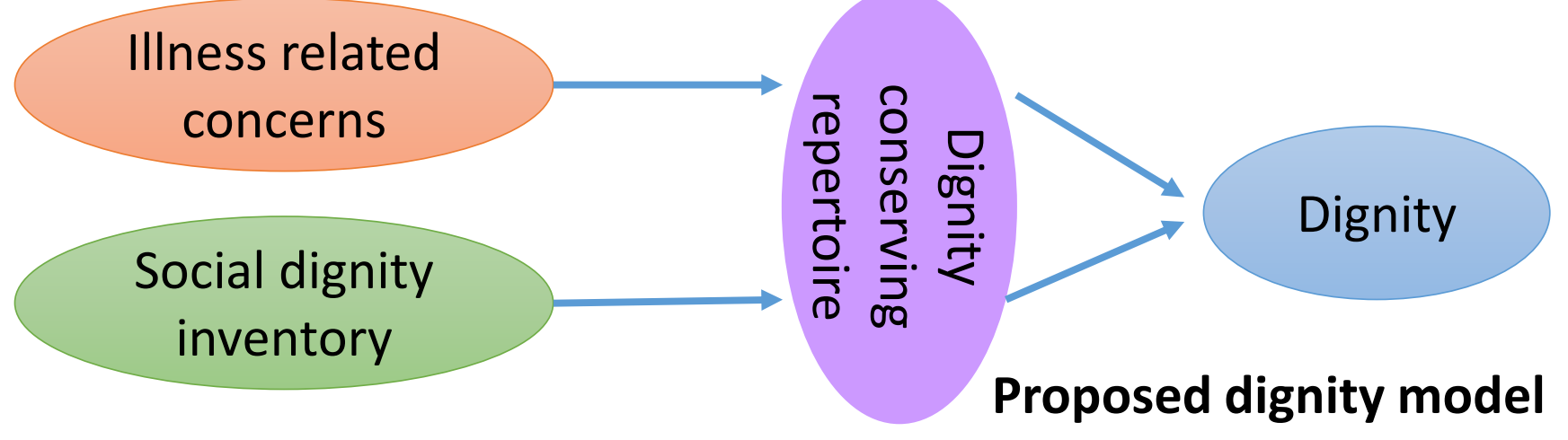

\begin{tabular}{|l|}
\hline CATEGORY \\
\hline Illness related concerns \\
(Issues related to illness itself \\
which threaten or impinge \\
on patients' sense of dignity) \\
\hline Dign
\end{tabular}

Dignity conserving repertoire

(One's worldview and attitude that helps to promote dignity or personal behavior that bolster dignity)

\begin{tabular}{|l|}
\hline THEME \\
\hline Level of independence \\
\hline Symptom distress
\end{tabular}

Dignity conserving perspective

Social dignity inventory (Social concerns or relationship dynamics that enhance or impinge on patients' sense of dignity)

Aftermath concerns

\section{SUBTHEMES}

Cognitive acuity

Functional capacity

Physical distress: death anxiety was not supported

Psychological distress

Role preservation: to preserve their role of a filial offspring or responsible parent

Generative/legacy: something lasting transcendent meant healthy and filial offspring, in other words, passing on filial piety from generation from generation.

Maintenance of pride: rather than the diminishing independence, patients feel loss of dignity when they are unable to maintain their sense of pride due to the impaired body image.

\begin{tabular}{l|l} 
Continuity of self & Hopefulness \\
\hline Autonomy/control & Acceptance
\end{tabular}

Resilience/fighting spirit

Living in the moment

Maintaining normalcy

Seeking spiritual comfort: spiritual comfort did not translate to specific religion in our study, but hold the belief of 'exerting the utmost of human abilities, and then resigning the rest to the mandate of Heaven', which in Chinese words of "Jin Ren Shi, Ting Tian Ming (尽人事，听天命)”。

\begin{tabular}{|l|l|} 
Privacy boundaries & Social support \\
\hline Care tenor & Burden to others
\end{tabular}

Communication openness: communication openness of information and emotions between patients and families.

Financial burden: unaffordable high medical expenditure which undermines patients' sense of dignity. 